\title{
The Science with the Interstellar Heliopause Probe
}

\author{
H. Fichtner ${ }^{1}$, B. Heber ${ }^{2}$, and M. Leipold ${ }^{3}$ \\ ${ }^{1}$ Institut für Theoretische Physik IV, Ruhr-Universität Bochum, 44780 Bochum, Germany \\ ${ }^{2}$ Institut für Experimentelle und Angewandte Physik, Christian-Albrechts-Universität Kiel 24118 Kiel, Germany \\ ${ }^{3}$ Kayser-Threde GmbH, Space Technology and Utilization Section, 81379 Munich, Germany
}

Received: 22 December 2005 - Revised: 14 May 2006 - Accepted: 19 May 2006 - Published: 15 June 2006

\begin{abstract}
After the exciting in-situ observations of the termination shock and the entry of the Voyager 1 spacecraft in the heliosheath, there is a growing awareness of the significance of the physics of the outer heliosphere. Its understanding helps to clarify the structure of our immediate interstellar neighbourhood, contributes to the clarification of fundamental astrophysical processes like the acceleration of charged particles at a steller wind termination shock, and also sheds light on the question to what extent interstellar-terrestrial relations are important for the environment of and on the Earth. Consequently, there are new seriously discussed suggestions for sending a modern spacecraft into the heliosheath and beyond. One of those candidates is the Interstellar Heliopause Probe (IHP) that has been studied in a Technology Reference Study by ESA/ESTEC. Here, we discuss the science objectives and expected scientific performance of this mission.
\end{abstract}

\section{Introduction}

After the first period of concept formulation regarding the large-scale structure of the solar cavity (Davis, 1955), nowadays called heliosphere according to a suggestion by Dessler (1967), a period of numerical modelling began in the late 1970's (Baranov et al., 1979) and is being continued since then, see the review by Zank (1999) and the book by Florinski et al. (2004a). The resulting 'heliospheric' paradigm claims that the heliosphere is a plasma bubble formed by the interaction of the solar wind with the local interstellar medium (LISM). It has a basic drop-like shape resulting from the relative motion of the Sun and the LISM as sketched in Fig. 1.

This paradigm has been corroborated by the direct or indirect observations:

(1) Most excitingly is the recent discovery of the solar wind

Correspondence to: H. Fichtner (hf@tp4.rub.de)

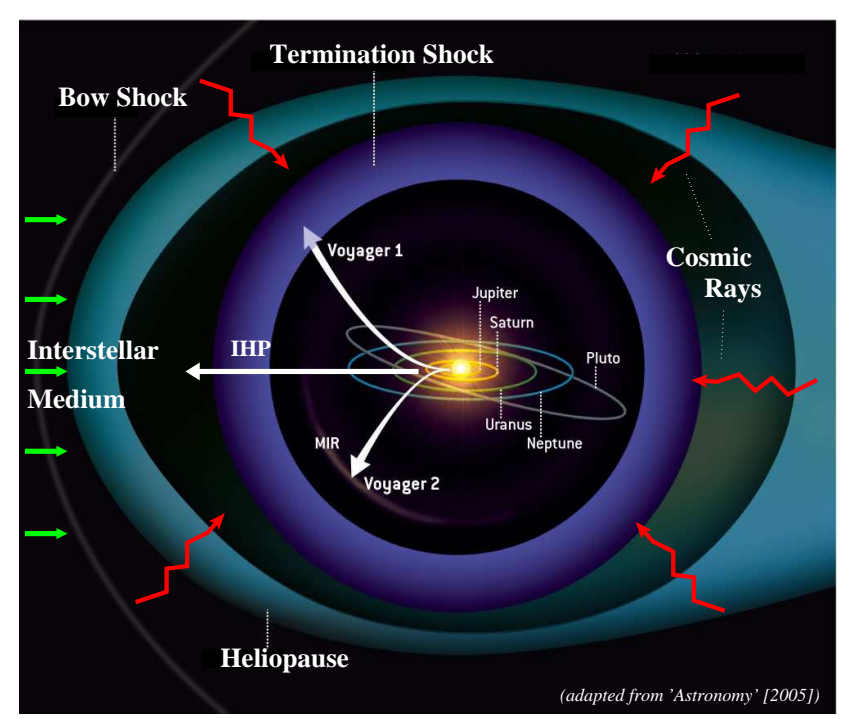

Fig. 1. A sketch of the structure of the heliopsheric interface (in the restframe of the Sun) and the trajectories of the Voyager spacecraft and the Interstellar Heliopause Explorer (IHP). The interface consists of three discontinuities, namely the termination shock where the solar wind is slowed from supersonic to subsonic speed, the heliopause separating the solar and the interstellar plasma, and the bow shock where the interstellar plasma possibly undergoes a transition to subsonic speed. In addition, there exists a hydrogen wall, i.e. a pile-up of neutral hydrogen between the heliopause and the bow shock as a consequence of its charge-exchange coupling to the interstellar plasma flow that is disturbed by the obstacle heliosphere (sketch adopted from Astronomy (2005)).

termination shock by Voyager 1 at $94 \mathrm{AU}$, see Burlaga et al. (2005), Decker et al. (2005), Gurnett and Kurth (2005), and Stone et al. (2005).

(2) Radio measurements with the two Voyager spacecraft (Gurnett et al., 2003; Kurth and Gurnett, 2003) can be

Published by Copernicus GmbH on behalf of the Arbeitsgemeinschaft Extraterrestrische Forschung e.V. 
interpreted as signatures from the interaction of a global merged interaction region (GMIR) with the heliopause Mitchell et al. (2004).

(3) There is overwhelming evidence from the analysis of interstellar absorption lines for the existence of a hydrogen wall ahead of the heliosphere (Linsky and Wood, 1996; Wood et al., 2004). Such hydrogen walls have been detected for other stars as well (Izmodenov et al., 1999; Wood et al., 2004).

(4) Some information about the local interstellar magnetic field appears to be available from measurements of starlight polarization and energetic particles, see Frisch (2003) and Gloeckler et al. (1997), respectively.

During recent years the study of the heliosphere as such has been extended towards its treatment as an astrosphere being embedded in a changing galactic environment. There is growing evidence that, in particular, cosmic rays are likely to play a role for the long-term conditions of the Earth's environment and on Earth itself (Shaviv, 2003; Shaviv and Veizer, 2003), and that the heliosphere serves to some extent as a protecting shield against the interstellar environment as reviewed by Scherer et al. (2002).

Parallel to the development of the theory of the heliosphere and its direct interstellar neighborhood, the LISM, space missions have shed light on many aspects of this paradigm that has emerged over the years. In addition to the measurement of the solar wind, the solar radiation back-scattered from neutral atoms, the direct detection of neutral atoms as well as so-called interstellar pick-up ions, especially the observations of cosmic rays have contributed to our present understanding, see the reviews by Fichtner (2001), Heber (2001), and Fichtner (2005).

Crucial for the further understanding is a direct in-situ observation of the heliospheric interface that can be defined as the region between the supersonically expanding solar wind and the (probably) supersonically streaming local interstellar medium (Fahr, 2000; Alexashov and Izmodenov, 2005). It consists of the inner heliosheath between the heliospheric shock terminating the supersonic expansion of the solar wind, the heliopause separating the solar and the interstellar plasma, and the outer heliosheath extending from the heliopause to the bow shock marking the transition of the interstellar plasma flow from supersonic to subsonic speed. The bow shocks of other stars have been detected (Brown and Bomans, 2005).

The only hope for an in-situ observation of the heliospheric interface in near future is based on the two Voyager spacecraft launched in the 1970's. With a power supply lasting for perhaps another 15 years these deep space probes will serve to explore the interface up to heliospheric distances of about 130-140 AU. This means that it is unclear whether or not they will observe the heliopause (named as such by Bhatnagar and Fahr (1972)) for which the distance estimates vary between about 110 and $160 \mathrm{AU}$ (Gurnett et al., 2003; Webber and Lockwood, 2004). In order to explore the structure and properties of the heliospheric interface it is, however, nec- essary to get in-situ observations from the heliopause and the region beyond. Therefore, a 'Heliopause Explorer' mission (Leipold et al., 2003), now called Interstellar Heliopause Probe (IHP, see Falkner (2005), Lyngvi et al. (2005a) and Lyngvi et al. (2005b)), can represent the next step in the exploration of the outer boundaries of the heliosphere.

\section{The scientific objectives of IHP}

\subsection{The basic questions}

An in-situ exploration of the heliopause and the heliospheric interface will allow us to answer a number of basic scientific questions that arose during the last 40 years of heliospheric research:

- What are the characteristics of the solar wind termination shock and the heliopause, where are these structures located and how do their characteristics and locations change with time?

- How does the solar wind termination shock act as an astrophysical accelerator of (anomalous) cosmic rays?

- How does the heliosphere shield the Earth from galactic cosmic rays and the interstellar neutral gas?

- What is the state and composition of the local interstellar medium beyond the heliopause?

In view of the recently revived interest in the so-called 'Pioneer Anomaly' (Anderson et al., 2002) a fifth question could be added:

- Is the 'Pioneer Anomaly' real and is Newton's law of gravitation to be modified?

As there is another mission being discussed in the context of this question (Nieto et al., 2005), we do not address it further here, but note that an extension of the scientific payload discussed below would allow to add a test of the Pioneer Anomaly to the scientific objectives.

Because all mentioned particle components, i.e. anomalous and galactic cosmic rays (ACRs, GCRs) and the interstellar gas are interacting with the heliospheric interface (Florinski et al., 2004b; Langner and Potgieter, 2005; Scherer and Ferreira, 2005) and are likely to influence the Earth's environment their understanding is of fundamental interest (Scherer et al., 2002). This interest manifests itself in the recent development of models that try to incorporate the kinetic cosmic ray transport into a dynamical description of the heliosphere, see, e.g. Florinski et al. (2003), Ferreira et al. (2004) or Scherer et al. (2004). Simulation examples are shown in Fig. 2. 

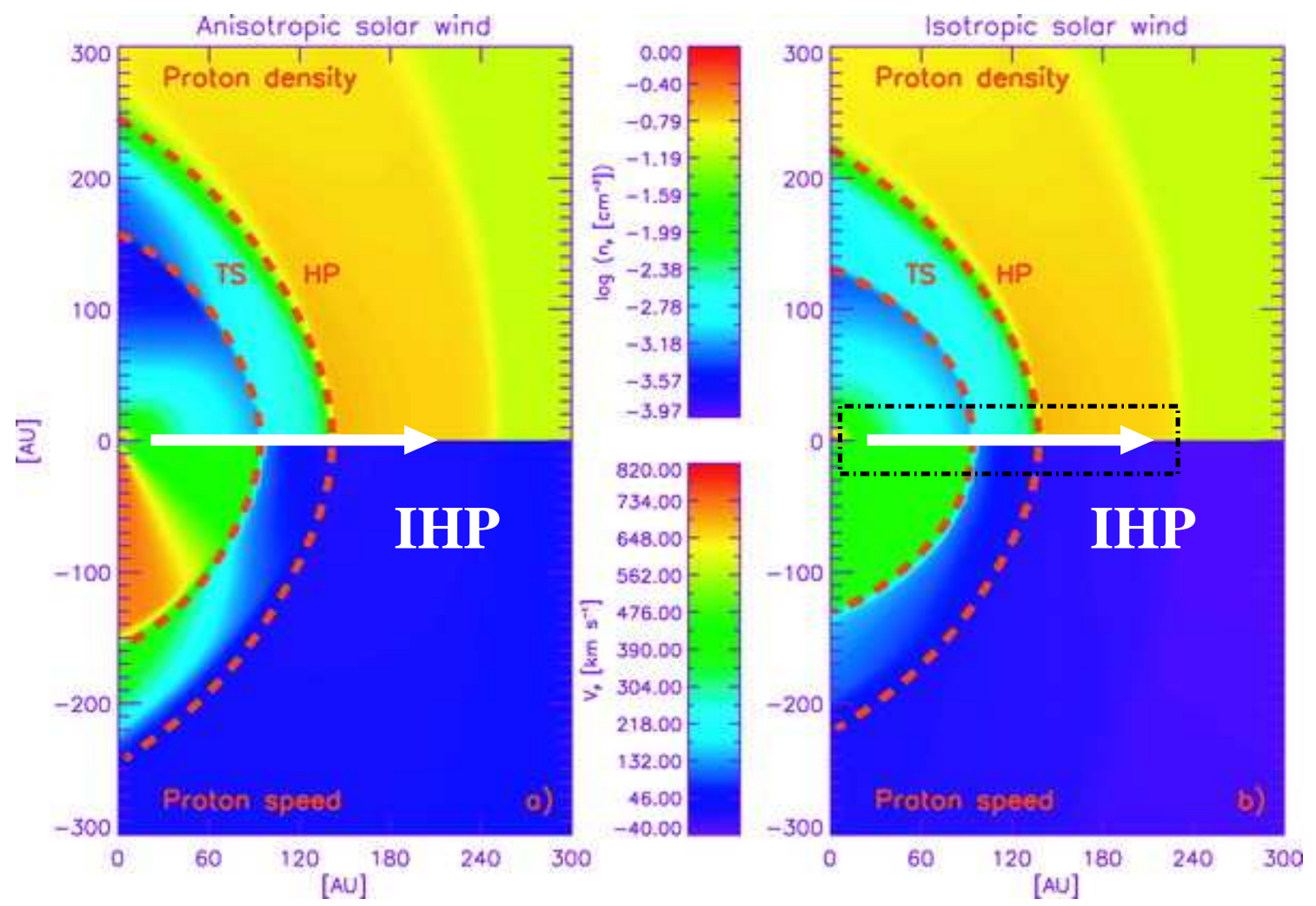

Fig. 2. The heliospheric proton density and speed as resulting from simulations with an (a) anisotropic and (b) isotropic solar wind, i.e. for solar minimum and maximum, respectively (Ferreira et al., 2004). The arrow sketches the trajectory of IHP and the box in the right panel indicates the region of interest for IHP. Note that the speed is always positive despite the colour scale extending to negative values.

\subsection{The discovery region}

The two simulations depicted in Fig. 2 are for solar minimum (left) and solar maximum (right) activity. The trajectory of IHP is at low heliographic latitudes, for which the resulting difference in heliospheric structure is expected to be of minor importance. Therefore, in what follows we limit the discussion to low latitudes as indicated by the box in the right panel, see also Fig. 4.

Despite the good progress that has been achieved with corresponding model simulations in the recent years, the applicability of their results remains limited due to the demanding computations. Further progress will benefit from better observational constraints.

Thus, in view of our limited knowledge of the heliospheric interface, the IHP will be a discovery mission that will provide valuable input to already existing models and will, thus, contribute tremendously to answer the questions formulated above.

\subsection{Relation to other missions}

The IHP is related to the only other seriously persued mission idea to explore the outer heliosphere and the LISM be- yond the heliopause in-situ, i.e. the NASA Interstellar Probe (Mewaldt and Liewer, 2001; Liewer et al., 2001), in the sense that IHP represents a moderately less ambitious but, at this stage, more realistic approach. More importantly, IHP is related to the Interstellar Boundary Explorer (IBEX, McComas et al. (2004)) that will map the outer heliosphere, in particular the heliosheath between the termination shock and the heliopause, by remote imaging of energetic neutral atoms (ENAs). The global heliospheric images, i.e. all-sky ENA flux maps resulting from remote observations with IBEX (for an example see Sternal et al. (2005)) and the in-situ measurements with IHP will be supplementary to each other.

\section{The scientific payload of IHP}

The above science objectives for an IHP mission determine a "strawman" instrument payload. Several technology developments especially in the miniaturization of the electronics and regarding modern light-weight materials are needed to enable the IHP to reach its goals. In order to fulfil the science objectives the following minimum instrumentation with challenging measurement requirements has to be considered: 


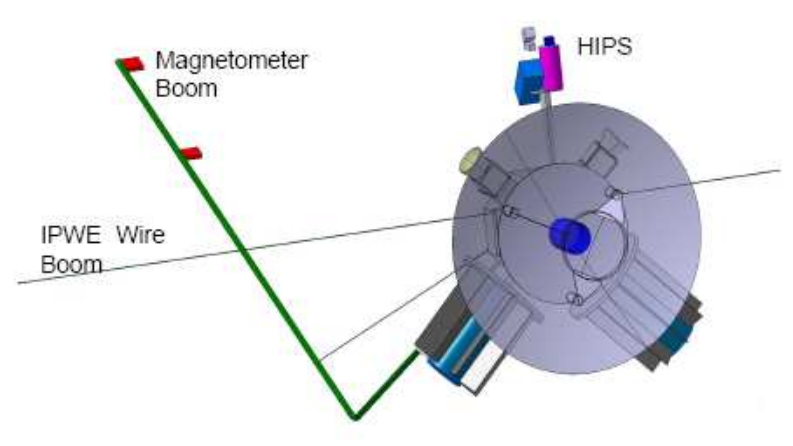

Fig. 3. A sketch of the IHP Science Payload accomodation on the spacecraft, showing the magnetometer and the IPWE wire boom (see Table 1) and the Highly Integrated Payload Suite (HIPS).

(1) A plasma analyzer needs to be able to map the solar wind plasma up to the termination shock, follow its thermalization in the region beyond and detect the transition from solar to interstellar plasma at the heliopause. Simultaneously, the instrumentation has to determine the composition and velocity distribution of the interstellar plasma.

(2) The purpose of a magnetometer is to map out the magnetic fields at the outer reaches of the heliosphere. The lowest magnetic field strengths are expected to be encountered just inside the termination shock and can be estimated to be of the order of $0.01 \mathrm{nT}$. In order to clearly resolve the direction of such a weak field it is necessary to have a magnetometer that is sensitive in each component down to $0.001 \mathrm{nT}$.

(3) An investigation of plasma and radio waves should have the following three high priority objectives. First, monitor the low-frequency heliospheric radio emissions. Second, survey plasma waves in the outer heliosphere and ISM beyond the heliopause. Third, provide an accurate, independent determination of the plasma density.

(4) An energetic particle detector consisting of an ion and an electron sensor will serve to study suprathermal pickup ions (PUIs), ACRs as well as GCRs, and to search for low energy cosmic ray electrons, which might be accelerated in the heliospheric interface. The ion sensor should cover the critical energy range above the plasma regime, where PUIs are accelerated out of the bulk distribution. The important objectives of the ion sensor are (i) the injection of PUIs at the termination shock and their acceleration into ACRs, (ii) the suprathermal extension of the heated solar wind and PUI distributions into the heliosheath, and (iii) the search for the low end of GCRs beyond the heliosphere.

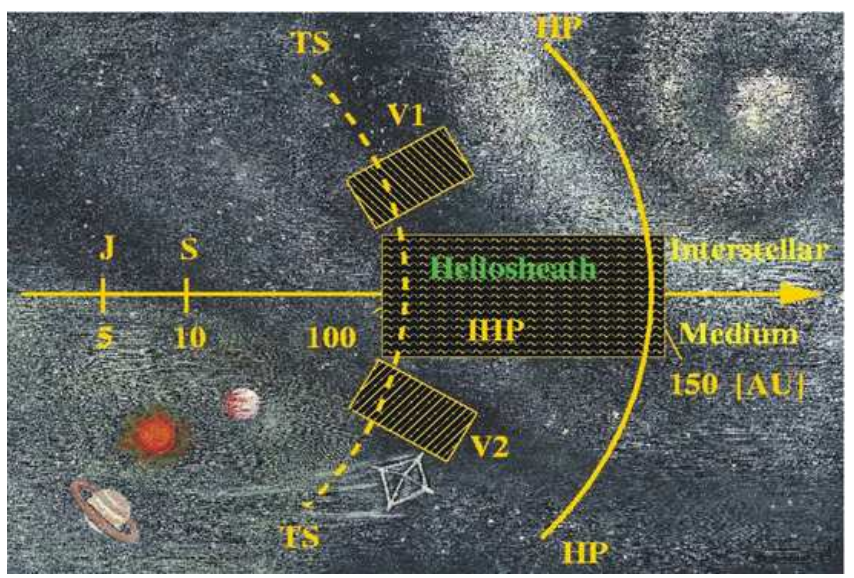

Fig. 4. Discovery region of the IHP as compared to those of the Voyager 1 and 2 spacecraft (IHP artwork by Indira Heber-Novkinić).

The primary instruments, along with their masses and power consumption are listed in Table 1.

In order to match the challenging requirements of accomodating payload on a small spacecraft, the approach of a Highly Integrated Payload Suite (HIPS) is used. With HIPS the payload is integrated as much as possible, sharing common functionalities like data processing, power supply, thermal and environmental control between the instruments. Maximum sharing of structures, optical benches, baffles and optics within physical limits is envisaged. This high integration of the instruments also allows for significant reduction of harness. Details about the technical aspects of the payload and its accomodation on the spacecraft (see Fig. 3) can be found in Lyngvi et al. (2005a) and Lyngvi et al. (2005b). Also the other more technical or spacecraft engineering matters such as the propulsion of the IHP for which a solar sail is discussed, the sail configuration, the trajectory and its implication for the thermal environment of IHP, are discussed elsewhere, see, e.g. Leipold et al. (2003), Leipold et al. (2005), and van den Berg et al. (2005).

\section{The scientific performance of IHP}

\subsection{General performance}

The success of the IHP mission will depend on the long-term performance of the scientific payload as described above. The major challenge consists in the measurement of the tenuous plasma environment including the magnetic field and plasma waves for at least 20 years so that IHP reaches heliocentric distances greater than 150-200 AU. The region to be explored by IHP is schematically shown in Fig. 4 along with those explored by the two Voyager missions.

As mentioned above Voyager 1 has encountered the solar wind termination shock on 16 December 2004, and Voy- 
Table 1. The scientific payload of IHP.

\begin{tabular}{lllc}
\hline Measurement objective & Instrument & $\begin{array}{l}\text { Mass } \\
{[\mathrm{kg}]}\end{array}$ & $\begin{array}{c}\text { Power } \\
{[\mathrm{W}]}\end{array}$ \\
\hline Solar Wind & Plasma Analyzer (IPA) & 2.0 & 1.3 \\
Plasma and Radio Waves & Plasma Wave and Radio Experiment (IPWE) & 4.5 & 4.0 \\
Magnetic Field & Magnetometer (IMAG) & 3.7 & 3.4 \\
Neutral Atoms & Neutral Atom Detector Imager (INCADI) & 0.5 & 1.8 \\
Energetic Particles & Energetic Particle Detector (IEPD) & 1.8 & 1.2 \\
Interstellar Dust & Dust Analyzer (IDA) & 1.0 & 1.0 \\
UV-Emission & UV-Photometer (IUVP) & 0.3 & 0.3 \\
Solar Monitor & Solar Activity Monitoring & 0.6 & 3.0 \\
\hline & Structures and Central Payload Power Supply & 3.0 & - \\
\hline & Margin 20\% & 3.5 & 3.2 \\
\hline Total & & $\mathbf{2 0 . 9}$ & $\mathbf{1 9 . 2}$ \\
\hline
\end{tabular}

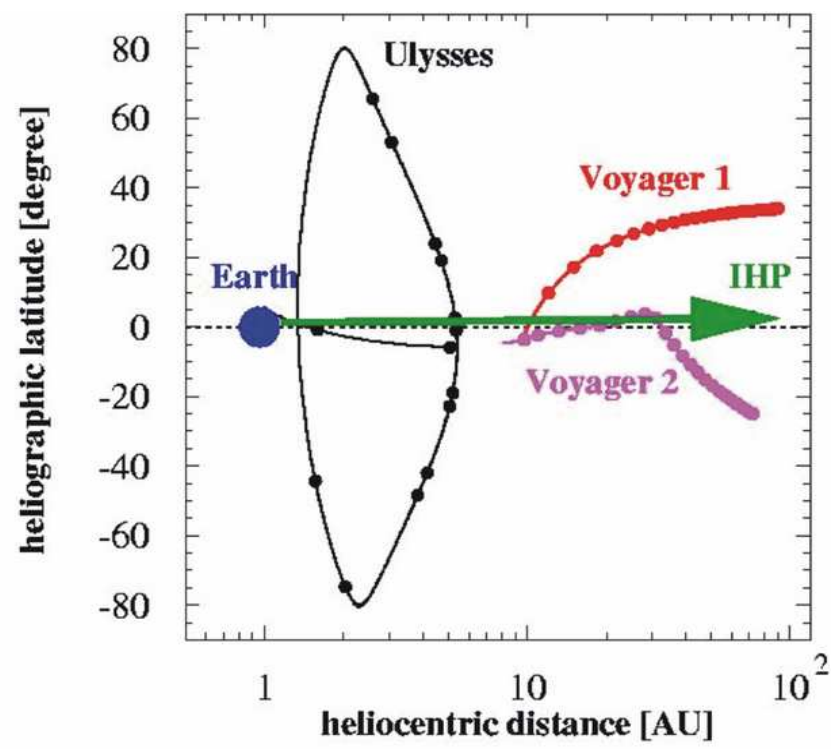

Fig. 5. The outer heliospheric network of spacecraft (adopted from Heber and Burger (1999)).

ager 2 can be expected to do so in 2007 or 2008, see Kiraly (2005). Given the nominal life-time of the power generator onboard, the Voyager spacecraft might not reach the heliopause, however. Although Voyager 1 will penetrate further into the heliosheath than Voyager 2, the information from the former spacecraft is limited because of a non-functional plasma instrument. IHP will extend the Voyager measurements in various ways. First, due to its different heliographic latitude it will explore the heliosheath near the ecliptic as sketched in Fig. 4.

Second, IHP will cross the heliosheath and be the first
1 AU Outer Heliosphere $(<130$ AU)

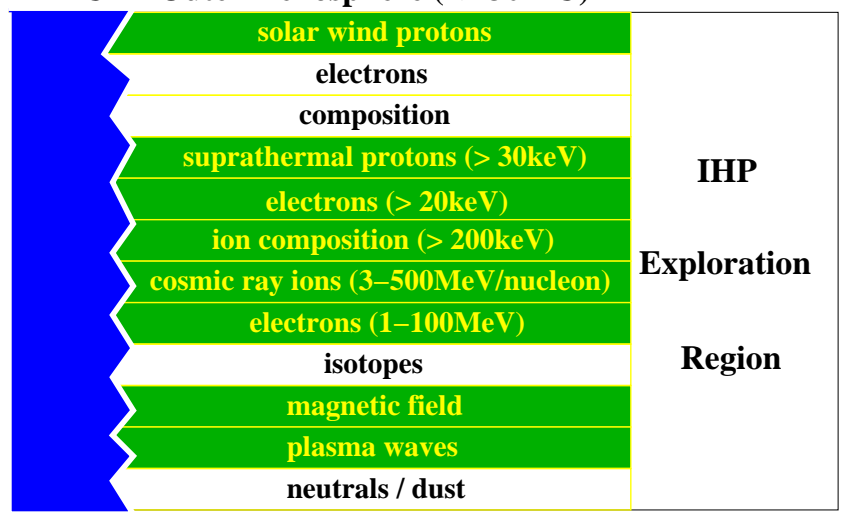

Fig. 6. The available measurements beyond $1 \mathrm{AU}$ and the extensions to be made with IHP up to $200 \mathrm{AU}$.

spacecraft to explore the heliopause and the boundary region towards the undisturbed local interstellar medium. IHP will, therefore, be an important cornerstone within the outer heliospheric network of spacecraft whose trajectories are schematically shown in Fig. 5.

Third, IHP will not only extend and improve the measurements made with the Voyager 1 and 2 spacecraft, but also observe the outer heliosphere at a different time. This will naturally enable us to get insight into the time dependence of the structure of the heliosphere.

\subsection{Specific performance of the scientific payload}

The instruments of the scientific payload are designed to measure in-situ the key quantities determining the structure and dynamics of the heliosphere as, for example, the solar 

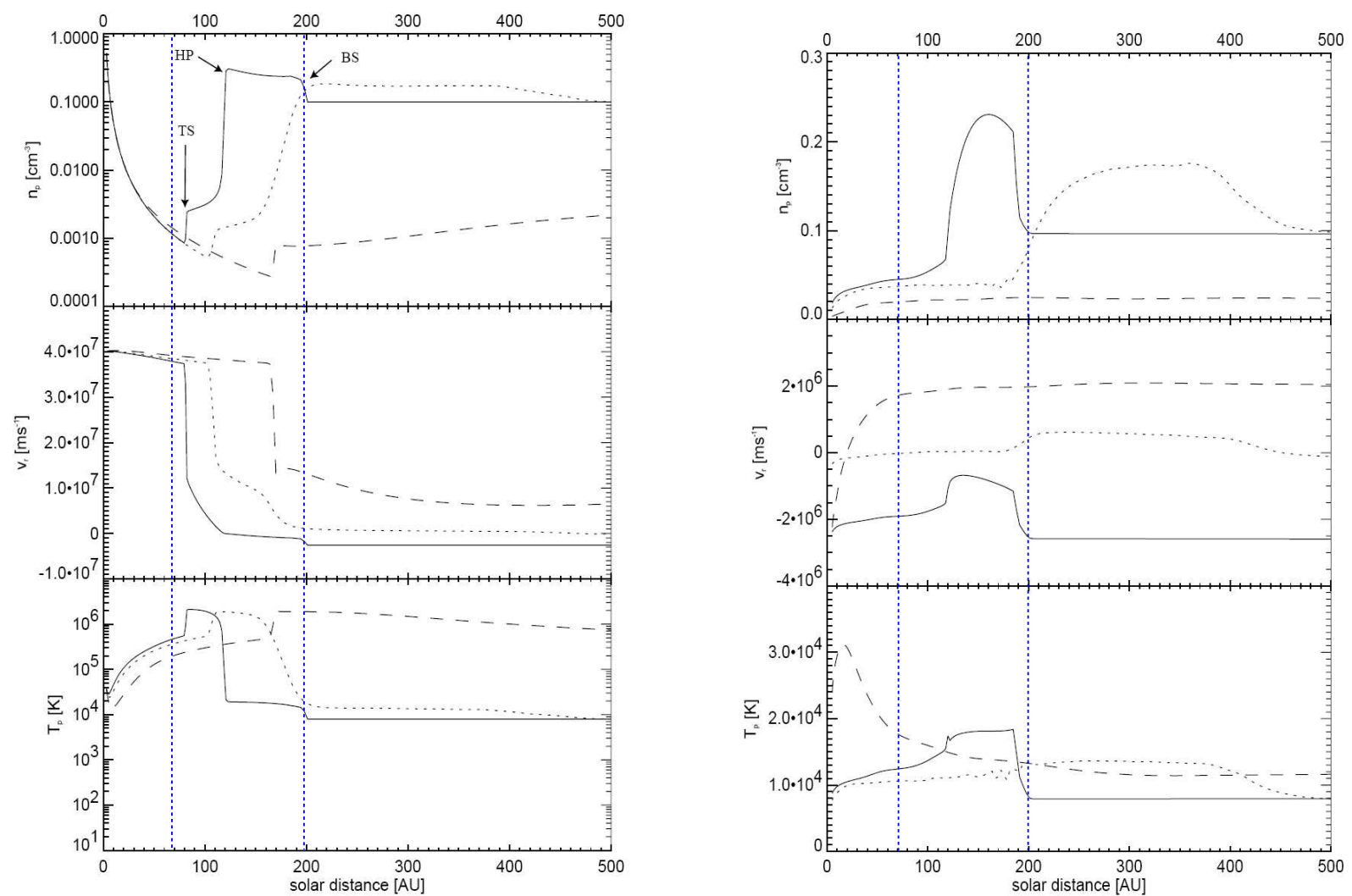

Fig. 7. Radial profiles of protons (left) and neutral hydrogen (right) in the upwind (solid), the crosswind (dotted), and the downwind (dashed) direction (Fahr et al., 2000). The vertical dashed lines indicate the main discovery region of IHP.

wind and interstellar plasma including the magnetic fields, the suprathermal and high-energy particles, as well as the neutral gas. Fig. 6 displays a schematic overview of available measurements close to the Earth and in the outer heliosphere including those to be made with the Voyagers out to about $130 \mathrm{AU}$.

While in the inner heliosphere a fleet of sophisticated spacecraft provides the listed quantitites in great detail, the existing measurements in the outer heliosphere are limited to certain particle species and energy ranges. Furthermore, the scientific payload of IHP is, unlike that of Voyager 1 and 2, designed to measure low magnetic field strengths, the composition, and complete energy range of suprathermal particles. This allows to answer the above-listed questions defining the IHP science objectives.

\subsubsection{Thermal particle populations}

For the first time not only the solar wind protons but also the solar wind electrons will be measured in the outer heliosphere, and the subsonic solar wind plasma will be observed up to the location of the heliopause. With IHP the first measurements of the (bow-shocked) interstellar plasma will be possible. This will enable us to confront our present under- standing of the physics of the heliosphere that is gained from model simulations (see, e.g. Fig. 7) with in-situ data.

While the Voyager spacecraft provide certain direct information about the plasma environment in the outer heliosphere, none is available regarding the neutral gas, and none will be available beyond about $130-140 \mathrm{AU}$, which is the distance range where the power supply onboard the Voyagers will become insufficient. The region around the heliopause is expected to be characteristically structured due to the charge exchange of interstellar neutral atoms with the solar wind, as displayed in the right panel of Fig. 7. The most prominent feature is the hydrogen wall (Baranov, 2002) located directly beyond the heliopause. Depending on the actual heliocentric distances of the heliopause (Gurnett et al., 2003; Webber and Lockwood, 2004) and the hydrogen wall, IHP will also explore the nature of the latter.

\subsubsection{Suprathermal particle populations}

Simultaneously, IHP will measure the spatial distribution and energy spectra of suprathermal particles as well as cosmic rays. Of special interest are the pick-up ions (PUIs), because they are directly originating from interstellar neutral atoms, influence the solar wind dynamics by mass and mo- 

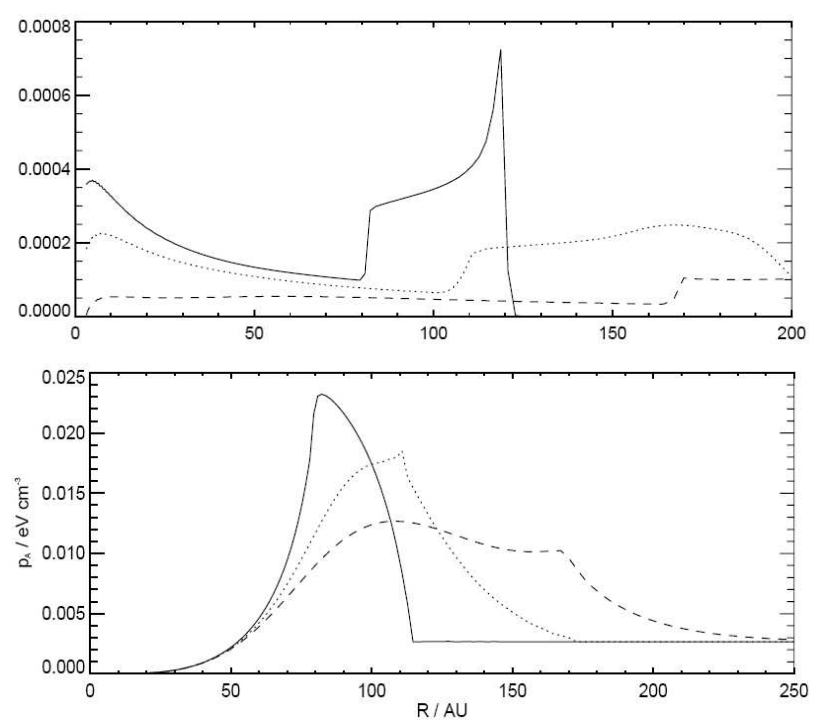

Fig. 8. Radial profiles of the energy density of pick-up protons (top) and anomalous cosmic rays (bottom) in the upwind (solid), the crosswind (dotted), and the downwind (dashed) direction (Fahr et al., 2000).

mentum loading in the outer heliosphere, and they represent the seed population of anomalous cosmic rays (ACRs). Their computed energy densities, given as functions of heliocentric distance in Fig. 8, can be compared directly with IHP data.

By extending the Voyager 1 and 2 measurements of energetic ions to intermediate energies (few keV) IHP will clarify the processes of pre-acceleration in the turbulent expanding solar wind and of the diffusive acceleration at the solar wind termination shock. Voyager observations as compared to model computations are shown in Fig. 9.

The upper curve in that figure is the model result for the proton spectrum at the termination shock and covers the whole range from solar wind to cosmic ray energies. Obviously, IHP measurements will tremendously enlarge the observed energy range towards lower energies and will thus provide crucial information. The Voyager observations were taken several tens of AU away from the source and are, therefore, subject to propagation effects. The computed lower curves in Fig. 9 show the modulation due to the turbulence in the magnetized solar wind. IHP supplements the particle observations with measurements of the rather weak magnetic fields $(>0.01 \mathrm{nT})$ in the outer heliosphere and is, thus, of prime importance regarding our knowledge about waveparticle interactions.

\subsubsection{Local interstellar spectra}

Present theory, modelling, and analyses of observations of galactic cosmic rays (Langner et al., 2001; Heber, 2004; Mewaldt et al., 2004; Potgieter and Langner, 2004) allow for

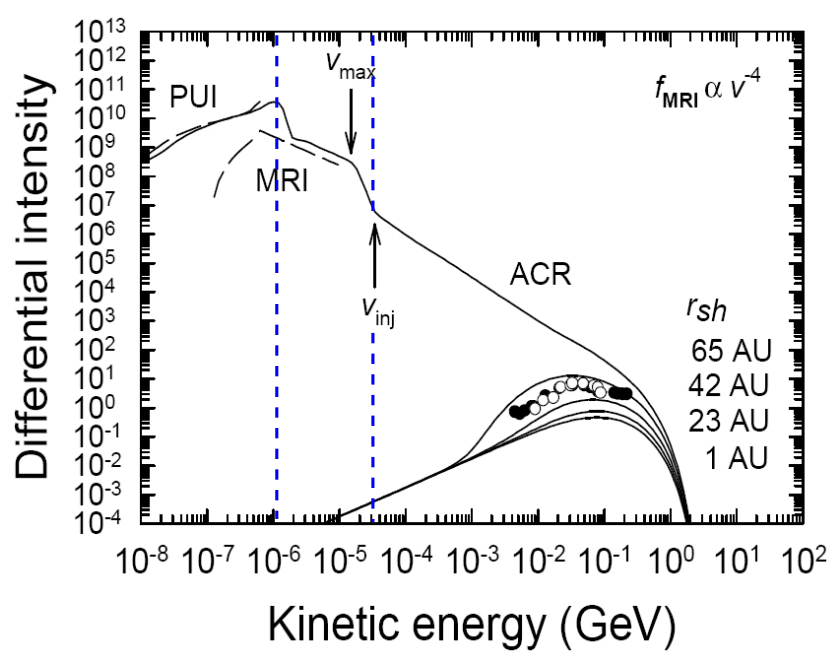

Fig. 9. Differential intensity in particles $/\left(\mathrm{m}^{2} \mathrm{~s}\right.$ sr MeV) of suprathermal particles pick-up ions, PUI; multiply reflected ions, MRI) together with that of ACRs. The symbols are Voyager observations of modulated ACR spectra (le Roux et al., 2000). The vertical dashed lines indicate the ion energy range that will become accessible via the plasma instrument of IHP.

a wide range of intensities of the interstellar spectra at low energies (see Fig. 10) that cannot be further narrowed without in-situ observations in the outer heliosphere. Also in this context, IHP will provide substantial information. It will be the first spacecraft to measure their unmodulated local interstellar energy spectra. Whether the latter are identical to the 'true' interstellar spectra upstream of the bow shock depends on the unknown nature of plasma turbulence between the heliopause and the bow shock, which IHP will measure as well.

\subsubsection{Plasma and radio waves}

Electron plasma oscillations are indicative for the upstream region of the termination shock as has been discussed by Gurnett and Kurth (2005) for Voyager 1's encounter with the shock on 16 December 2004. IHP will clarify whether such oscillations exist also near the heliopause and the bow shock, as one should expect from in analogy to planetary bow shocks. IHP should also locate the actual source region of the kHz-radio emission supposedly in the vicinity of the heliopause, for a review see Czechowski (2004).

These observations will help to understand the question of the stability of the heliopause as discussed, e.g. by Baranov et al. (1992), Liewer et al. (1996), Wang and Belcher (1999), Ruderman (2000), Opher at al. (2003), and Florinski et al. (2005). 


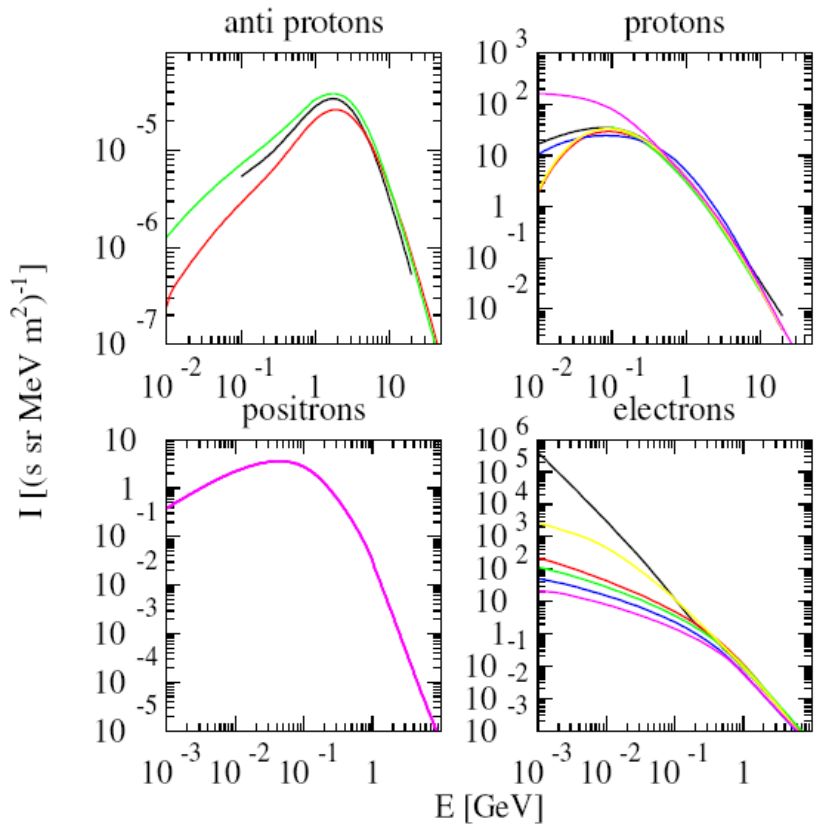

Fig. 10. Computed local interstellar spectra of protons and electrons (Heber, 2001).

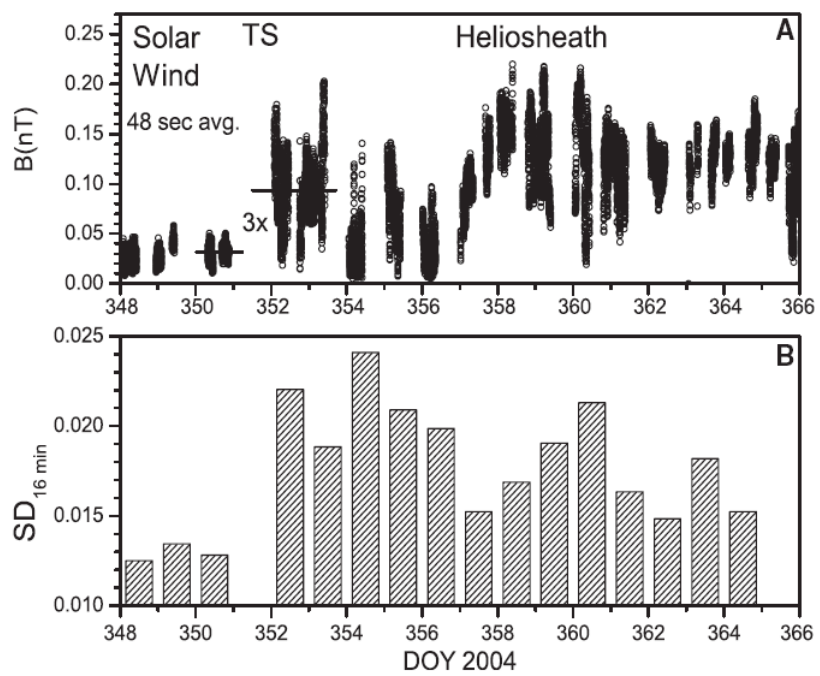

Fig. 11. 48 second-averaged magnetic field strength $B$ (nT) before and after the termination shock crossing of Voyager 1 and measurements of daily averaged higher frequency fluctuations up to $0.25 \mathrm{~Hz}$ over 16 min intervals ( $S D_{16 \mathrm{~min}}$ ), see Burlaga et al. (2005).

\subsubsection{Magnetic field}

Very interesting will be the measurements of the magnetic field in the heliosheath and beyond. The first Voyager results show, as expected, a compression of the field by a fac-

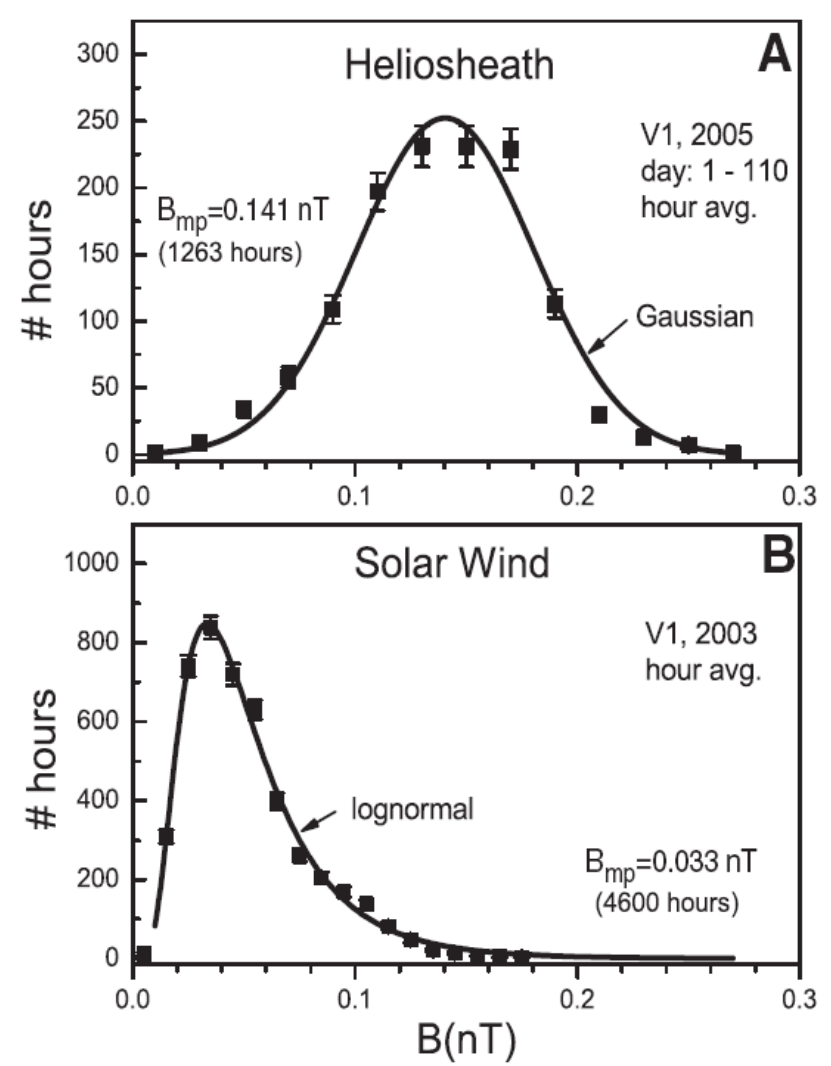

Fig. 12. The statistical distribution of the observed magnetic field strength (upper panel) in the heliosheath and (lower panel) in the upstream solar wind, see Burlaga et al. (2005).

tor of about three (Burlaga et al., 2005), see the upper panel of Fig. 11.

Interestingly, however, no sector boundaries were observed in the heliosheath during the first few months after the shock encounter, giving support to the idea of a low convection speed of the order of the spacecraft speed of ca. $17 \mathrm{~km} / \mathrm{s}$ (Decker et al., 2005). Further evidence for a significantly altered magnetic field in the downstream region comes from its fluctuations, which are much stronger in the heliosheath, see lower panel of Fig. 11. Finally, also the statistical distribution of field magnitude changed from lognormal (upstream) to gaussian (heliosheath), see Fig. 12.

All of these measurements will be repeated with higher precission with the IHP magnetometer that will be, in contrast to those on the Voyagers, designed to measure such weak magnetic fields. In addition, IHP will measure the magnetic field beyond the heliopause whose strength, turbulence state and exact orientation are unknown. Thereby it can provide important constraints for the magnetohydrodynamic modelling of the heliosphere that began with the work by Fujimoto and Matsuda (1991). The subsequent work is reviewed in Zank (1999), and nearly all contemporary mod- 
elling efforts are contained in Florinski et al. (2004a). An example for younger ideas is the possible reconnection of the heliospheric and interstellar magnetic field as described in Nickeler and Fahr (2005).

\subsubsection{Dust and ultraviolet radiation, solar monitoring}

IHP measurements of dust beyond $20 \mathrm{AU}$ will show what the contribution of Kuiper-belt objects to the dust population is (e.g. Moro-Martín and Malhotra (2002)) and, upon approaching the heliopause, will reveal to what extent heliospheric magnetic fields can hinder dust particles to penetrate into the inner heliosphere, see e.g. Czechowski and Mann (2003).

The observations of ultraviolet radiation with IHP will supplement the in-situ measurements and allow to derive information about regions far away from the spacecraft as has been done for the Voyager mission (Murthy et al., 1999). The combination of all data will lead to an approximation to the global structure of the heliosphere.

Finally, there is an instrument for the monitoring of solar activity.

\section{Conclusions}

The Interstellar Heliopause Probe (IHP) will fundamentally contribute to our understanding of heliospheric physics and astrophysics. In particular, by answering the questions formulated above it will reveal the structure of the heliosphere as an example of an astrosphere, it will clarify the significance and efficiency of astrophysical acceleration processes, it will probe the cosmic ray modulation in the heliosheath and contribute to the understanding of interstellar-terrestrial relations, it will identify the state and compositon of the local interstellar medium as a representative of the general interstellar medium. Obviously, as these topics are of interest to solar physics, plasma physics and astrophysics, IHP will be a true interdisciplinary discovery mission.

Acknowledgements. H.F. is very grateful to his most important supervisor, Hans-Jörg Fahr, who was honoured with the workshop 'Future Perspectives in Heliospheric Research' held on the occasion of his 65th birthday. H.F. also thanks the staff of the Physikzentrum Bad Honnef for providing an excellent environment, in which it was easy to have stimulating and very interesting discussions.

The IHP Technology Reference Study was performed under funding of ESA/ESTEC SCI-A, with Kayser-Threde as industrial prime contractor. The authors would like to acknowledge the support and contributions by Dr. P. Falkner, A. Lyngvi, Dr. S. Kraft, COSINE research, for the payload definition.

Edited by: H.-J. Fahr

Reviewed by: S. Grzedzielski and another referee

\section{References}

Alexashov, D. and Izmodenov, V.: Kinetic vs. multi-fluid models of $\mathrm{H}$ atoms in the heliospheric interface: a comparison, Astron. Astrophys., 439, 1171-1181, 2005

Anderson, J., Laing, P. A., Lau, E. L., Liu, A. S., Nieto, M. M., and Turyshev, S. G.: Study of the anomalous acceleration of Pioneer 10 and 11, Phys. Rev., D 65, 082004-1-50, 2002.

adopted from: http://voyager.jpl.nasa.gov/news/tenthop.html, Astronomy magazine, February 2005.

Baranov, V. B.: The heliosheath as a special case of stellarsheaths and the hydrogen wall as a signature of the heliosheath, Planet. Space Sci., 50, 535-539, 2002.

Baranov, V. B., Lebedev, M. G. and Ruderman, M. S.: Structure of the region of solar wind - Interstellar medium interaction and its influence on $\mathrm{H}$ atoms penetrating the solar wind, Astrophys. Space Sci., 66, 441-451, 1979.

Baranov, V. B., Fahr, H.-J., and Ruderman, M. S.: Investigation of macroscopic instabilities at the heliopause boundary surface, Astron. Astrophys., 261, 341-347, 1992.

Bhatnagar, V. P. and Fahr, H.-J.: Solar wind expansion beyond the heliosphere, Planet. Space Sci., 20, 445-460, 1972.

Brown, D. and Bomans, D. J.: To see or not to see a bow shock Identifying bow shocks with $\mathrm{H} \alpha$ allsky surveys, Astron. Astrophys., 439, 183-194, 2005

Burlaga, L. F., Ness, N. F., Acuna, M. H., Lepping, R. P., Connerney, J. E. P., Stone, E. C., and McDonald, F. B.: Crossing the Termination Shock into the Heliosheath: Magnetic Fields, Science, 309, 2027-2029, 2005.

Czechowski, A.,: Remote sensing of the heliospheric boundary, Adv. Space Res., 34, 79-87, 2004.

Czechowski, A. and Mann, I.: Local interstellar cloud grains outside the heliopause, Astron. Astrophys., 410, 165-173, 2003.

Davis, L. E.: Interplanetary magnetic fields and cosmic rays, Phys. Rev., 100, 1440-1444, 1955.

Decker, R. B., Krimigis, S. M., Roelof, E. C., Hill, M. E., Armstrong, T. P., Gloeckler, G., Hamilton, D. C., and Lanzerotti, L. J.: Voyager 1 in the Foreshock, Termination Shock, and Heliosheath, 309, 2020-2024, 2005.

Dessler, A. J.: Solar wind and interplanetary field, Rev. Geophys., 5, 1-41, 1967.

Fahr, H.-J.: The Multifluid Character of the 'Baranov' Interface, Astrophys. Space Sci., 274, 35-54, 2000.

Fahr, H.-J., Kausch, T., and Scherer, H.: A 5-fluid hydrodynamic approach to model the solar system-interstellar medium interaction, Astron. Astrophys., 357, 268-282, 2000.

Falkner, P.: Update on ESA's Technology Reference Studies, 56th Int. Astronautical Congress 2005, IAC-05-A3.4.01, Fukuoka, Japan, Oct 17-21, 2005.

Ferreira, S. E. S., Potgieter, M. S., and Scherer, K.: Modulation of Cosmic-Ray Electrons in a Nonspherical and Irregular Heliosphere, Astrophys. J., 607, 1014-1023, 2004.

Fichtner, H.: Anomalous Cosmic Rays: Messengers from the Outer Heliosphere, Space Sci. Rev., 95, 639-754, 2001.

Fichtner, H.: Cosmic Rays in the Heliosphere: Progress in the Modelling during the past ten years, Adv. Space Res., 35, 512-517, 2005.

Florinski, V., Zank, G. P., and Pogorelov, N. V.: Galactic cosmic ray transport in the global heliosphere, J. Geophys. Res., 108, 1228, doi:10.1029/2002JA009695, SSH 1-1-SSH 1-16, 2003. 
Physics of the Outer Heliosphere, AIP Conference Proceedings, 719, held 8-13 February, 2004 in Riverside, California, edited by Florinski, V., Pogorelov, N. V., and Zank, G. P. Melville, NY: American Institute of Physics, 2004a.

Florinski, V., Zank, G. P., Jokipii, J. R., Stone, E. C., and Cummings, A. C.: Do Anomalous Cosmic Rays Modify the Termination Shock? Astrophys. J., 610, 1169-1181, 2004b.

Florinski, V., Zank, G. P., and Pogorelov, N. V.: Heliopause stability in the presence of neutral atoms: Rayleigh-Taylor dispersion analysis and axisymmetric MHD simulations, J. Geophys. Res., 110, CiteID A07104, DOI 10.1029/2004JA010879, 2005.

Frisch, P. C.: Boundary conditions of the heliosphere, J. Geophys. Res., 108, LIS 11-1, CiteID 8036, DOI 10.1029/2003JA009909, 2003.

Fujimoto, Y. and Matsuda, T.: Interaction between the solar wind and magnetized local interstellar medium, preprint No. KUGD91-2, Kyoto University, 1-5, 1991.

Gloeckler, G., Fisk, L. A., and Geiss, J.: Anomalously small magnetic field in the local interstellar cloud, Nature, 386, 374-377, 1997.

Gurnett, D. A., Kurth, W. S., and Stone, E. C.: The return of the heliospheric 2-3 kHz radio emission during solar cycle 23 , Geophys. Res. Lett., 30, 2209, doi:10.1029/2003GL018514, 2003.

Gurnett, D. A. and Kurth, W. S.: Electron Plasma Oscillations Upstream of the Solar Wind Termination Shock, Science, 309, 2025-2027, 2005.

Heber, B.: Galactic and anomalous cosmic rays in the heliosphere, 27th Int. Cosmic Ray Conf., Hamburg, Highlight and Rapporteur Papers, 118-135, 2001

Heber, B.: Propagation and modulation of cosmic rays in the heliosphere, Plasmas in the laboratory and in the universe: New Insights and New Challenges, AIP Conf. Proc., 703, 111-122, 2004.

Heber, B. and Burger, R. A.: Modulation of galactic cosmic rays at solar minimum, Space Sci. Rev., 89, 125-138, 1999.

Izmodenov, V. V., Lallement, R., and Malama, Y. G.: Heliospheric and it astrospheric hydrogen absorption towards Sirius: no need for interstellar hot gas, Astron. Astrophys., 342, L13-L16, 1999.

Kiraly, P.: The way out of the Bubble: implications of recent Voyager-1 data, 29th Proc. Int. Cosmic Ray Conf., 3-10 August, 2005, Pune, India, Session, SH 2.4, in press.

Kurth, W. S. and Gurnett, D. A.: On the source location of lowfrequency heliospheric radio emissions, J. Geophys. Res., 108, LIS 2-1, CiteID 8027, DOI 10.1029/2003JA009860, 2003.

Langner, U. W. and Potgieter, M. S.: The heliospheric modulation of cosmic ray protons during increased solar activity: effects of the position of the solar wind termination shock and of the heliopause, Ann. Geophys., 23, 1499-1504, 2005, mboxhttp://www.ann-geophys.net/23/1499/2005/.

Langner, U. W., de Jager, O. C., and Potgieter, M. S.: On the local interstellar spectrum for cosmic ray electrons, Adv. Space Res., 27, 517-522, 2001.

Leipold, M., Fichtner, H., Heber, B., Groepper, P., Lascar, S., Burger, F., Eiden, M., Niederstadt, T., Sickinger, C., Herbeck, L., Dachwald, B., and Seboldt, W.: Heliopause Explorer - a sailcraft mission to the outer boundaries of the solar system, in: Proc. Fifth IAA International Conference on Low-Cost Planetary Missions, 24-26 September 2003, Noordwijk, The Netherlands, Compiled by R. A. Harris. ESA SP-542, 367-375, 2003.
Leipold, M., Lyngvi, A., Falkner, P., Lappas, V., Fichtner, H., Kraft, S., and Heber, B.: Interstellar Helipopause Probe - Design of a Challenging Mission to 200 AU, in: Proc. 39th ESLAB Symposium, edited by Favata, F., Gimenez, Noordwijk, The Netherlands, April 19-21, 2005.

le Roux, J. A., Zank, G. P., Fichtner, H., and Ptuskin, V.: Selfconsistent injection and acceleration of pickup ions at the solar wind termination shock, Geophys. Res. Lett., 27, 2873, 2000.

Liewer, P. C., Karmesin, S. R., and Brackbill, J. U.: Hydrodynamic instability of the heliopause driven by plasma-neutral chargeexchange interactions, J. Geophys. Res., 101, 17 119-17 128, 1996.

Liewer, P. C., Mewaldt, R. A., Ayon, J. A., Garner, C., Gavit, S., and Wallace, R. A.: Interstellar probe using a solar sail: Conceptual design and technological challenges, in: The Outer Heliosphere: The Next Frontiers, edited by Scherer, K., Fichtner, H., Fahr, H.J., and Marsch, E., Pergamon Press (Amsterdam), COSPAR Colloquiua Series, 11, 401-420, 2001.

Linsky, J. L. and Wood, B. E.: The alpha Centauri Line of Sight: D/H Ratio, Physical Properties of Local Interstellar Gas, and Measurement of Heated Hydrogen (The 'Hydrogen Wall') Near the Heliopause, Astrophys. J., 463, 254-270, 1996.

Lyngvi, A., Falkner, P., Kemble, S., Leipold, M., and Peacock, A.: The interstellar heliopause probe, Acta Astron., 57, 104-111, 2005.

Lyngvi, A., Falkner, P., and Peacock, A.: The interstellar heliopause probe technology reference study, Adv. Space Res., 35, 20732077, 2005.

McComas, D., Allegrini, F., Bochsler, P., Bzowski, M., Collier, M., Fahr, H.-J., Fichtner, H., Frisch, P., Funsten, H., Fuselier, S., Gloeckler, G., Gruntman, M., Izmodenov, V., Knappenberger, P., Lee, M., Livi, S., Mitchell, D., Mbius, E., Moore, T., Reisenfeld, D., Roelof, E., Schwadron, N., Wieser, M., Witte, M., Wurz, P., and Zank, G. P.: The Interstellar Boundary Explorer (IBEX), in: Physics of the Outer Heliosphere, AIP Conference Proceedings, 719, held 8-13 February, 2004 in Riverside, California, edited by Florinski, V., Pogorelov, N. V., and Zank, G. P., Melville, NY: American Institute of Physics, 162-181, 2004.

Mewaldt, R. A. and Liewer, P. C.: Scientific Payload for an Interstellar Probe Mission, in: The Outer Heliosphere: The Next Frontiers, edited by Scherer, K., Fichtner, H., Fahr, H.-J., and Marsch, E., Pergamon Press (Amsterdam), COSPAR Colloquiua Series, 11, 451-464, 2001.

Mewaldt, R. A., Wiedenbeck, M. E., Scott, L. M., Binns, W. R., Cummings, A. C., Davis, A. J., Israel, M. H., Leske, R. A., Stone, E. C., and von Rosenvinge, T. T.: Cosmic-Ray Spectra in Interstellar Space, in: Physics of the Outer Heliosphere, AIP Conference Proceedings, 719, held 8-13 February, 2004 in Riverside, California, edited by Florinski, V., Pogorelov, N. V., and Zank, G. P., Melville, NY: American Institute of Physics, 127-132, 2004.

Mitchell, J. J., Cairns, I. H., and Robinson, P. A.: Theory for 2$3 \mathrm{kHz}$ radiation from the outer heliosphere, J. Geophys. Res., 109, CiteID A06108, 2004.

Moro-Martin, A. and Malhotra, R.: A Study of the Dynamics of Dust from the Kuiper Belt: Spatial Distribution and Spectral Energy Distribution, Astron. J., 124, 2305-2321, 2002.

Murthy, J., Hall, D., Earl, M., Henry, R. C., and Holberg, J. B.: An Analysis of 17 Years of Voyager Observations of the Diffuse Far- 
Ultraviolet Radiation Field, Astrophys. J., 522, 904-914, 1999.

Nickeler, D. and Fahr, H.-J.: Reconnection at the heliopause, Adv. Space Res., 35, 2067-2072, 2005.

Nieto, M. M., Turyshev, S. G., and Anderson, J. D.: The Pioneer Anomaly: The Data, its Meaning, and a Future Test, in: Gravitation and Cosmology: 2nd Mexican Meeting on Mathematical and Experimental Physics, AIP Conference Proceedings 758, 113-128, 2005.

Opher, M., Liewer, P. C., Gombosi, T., Manchester, W., deZeeuw, D. L., Sokolov, I., and Toth, G.: Probing the Edge of the Solar System: Formation of an Unstable Jet-Sheet, Astrophys. J., 529, L61-65, doi: 10.1086/376960, 2003.

Potgieter, M. and Langner, U.: The heliospheric modulation of cosmic ray boron and carbon, Ann. Geophys., 22, 3729-3740, 2004, mboxhttp://www.ann-geophys.net/22/3729/2004/.

Ruderman, M. S.: Absolute and Convective Instability of Tangential Discontinuities in Viscous Fluids: Application to Heliopause, Astrophys. Space Sci., 274, 327-341, 2000.

Scherer, K., Fichtner, H., and Stawicki, O.: Shielded by the wind: the influence of the interstellar medium on the environment of Earth, J. Atm. Sol.-Terr. Phys., 64, 795-804, 2002.

Scherer, K., Fahr, H.-J., Fichtner, H., and Heber, B.: Long-Term Modulation of Cosmic Rays in the Heliosphere and its Influence at Earth, Sol. Phys., 224, 305-316, 2004.

Scherer, K. and Ferreira, S. E. S.: A heliospheric hybrid model: hydrodynamic plasma flow and kinetic cosmic ray transport, ASTRA, 1, 17-27, 2005.

Shaviv, N.: The spiral structure of the Milky Way, cosmic rays, and ice age epochs on Earth, New Astron,. 8, 1, 39-77, 2003.

Shaviv, N. and Veizer, J.: Celestial Driver of Phanerozoic Climate?,
GSA Today, 13, 4-10, 2003.

Sternal, O., Scherer, K., Fichtner, H., and Fahr, H.-J.: All-sky ENA flux maps for IBEX from 3D modelling, Proc. 11th Solar Wind Conf., 12-17 June, 2005, Whistler, Canada, in press, 2005.

Stone, E. C., Cummings, A. C., McDonald, F. B., Heikkila, B. C., Lal, N., and Webberm, W. R.: Voyager 1 Explores the Termination Shock Region and the Heliosheath Beyond, Science, 309, 2017-2020, 2005.

van den Berg M. L., Lyngvi, A., Falkner, P., Kemble, S., Leipold, M., Kraft, S., and Peacock, A.: Interstellar Heliopause Probe - An ESA Technology Reference Study, EGU 2005, Vienna, Austria, April 24-29, 2005.

Wang, C. and Belcher, J. W.: The heliospheric boundary response to large-scale solar wind fluctuations: a gasdynamic model with pick-up ions, J. Geophys. Res., 104, 549-556, 1999.

Webber, W. R. and Lockwood, J. A.: Large global transient decreases of $>70 \mathrm{MeV}$ cosmic rays observed at Voyager 1 and Voyager 2 in the outer heliosphere in 2001-2003: The onset of heliospheric 2-3 kHz radio emission and the distance to the heliopause, J. Geophys. Res., 109, CiteID A06107, DOI 10.1029/2003JA010340, 2004.

Wood, B. E., Müller, H. R., Tank, G. P., Izmodenov, V. V., and Linsky, J. L.: The heliospheric hydrogen wall and astrospheres, Adv. Space Res., 34, 66-73, 2004.

Zank, G. P.: Interaction of the solar wind with the local interstellar medium: a theoretical perspective, Space Science Reviews, 89, 413-688, 1999. 